\title{
EDITORIAL
}

\section{Changing mindsets}

\author{
Beyond the laboratory bench, cancer researchers will face big challenges during their careers. \\ Nature Reviews Cancer has published two Viewpoint articles to highlight some of these wider issues.
}

A career in cancer research is filled with demands and challenges. Besides the need for producing high-quality research, establishing collaborations and securing funding, this career often includes a lack of job security, a poor career structure and difficult work culture. Navigation of this career path is easier when there is support and guidance at hand, especially for younger research scientists. However, the parameters of scientific success are to a large degree still numerical, and the committed training and mentoring of the next generation of scientists are not included in the equations.

In our September issue, we published a Viewpoint ${ }^{1}$ to highlight the benefits that effective mentoring brings to cancer research. We asked three recipients of the Nature Awards for Mentoring in Science to discuss what great mentorship means to them and how it can contribute to improving the scientific landscape. Although effective mentoring often comes with a major time commitment, it is time well spent on helping mentees to align their goals and career paths according to their individual strengths and interests, and encouraging openness and confidence in doing research, in which overcoming perceived failure is an essential part of the process. This spirit of communication and support will shape relationships in teams and help to create a more positive and inclusive work environment in research.

A common mindset is that good mentoring is a non-essential skill in climbing the career ladder. This mindset disadvantages those who are committed to mentoring and is worsened by the gender-biased view of mentoring as a task that comes easier to women than men. Group leaders are often underequipped with the skills and/or dedication to provide such training to their teams. To change this, institutions and funders can do their part by formally recognizing that to advance cancer research, the well-rounded training of young scientists as well as group leaders is essential.

The second Viewpoint ${ }^{2}$, in our October issue, came about from our participation in a 'Women in Science Mentoring' panel that took place for the first time earlier this year at the Lorne Cancer conference in Australia. It is clear that gender inequality remains a challenge in science, despite considerable progress and recognition. Unfortunately, it will be one that many early career female scientists entering the field of cancer research will face. To highlight this problem with our readers, we asked the four female researchers who were also part of the panel to discuss their inspiring career stories and outline what they believe to be potential solutions. The causes and consequences of gender bias in the scientific community are hard to pinpoint, but perhaps the take-home message from our Viewpoint article is the need for institutions to foster a culture of positive empowerment for women, providing opportunities for advancement and leadership, whilst funding bodies and scientific journals should be providing increased exposure and encouraging more submissions from female scientists.

In our journal, we are keen to right the balance by actively taking measures to achieve diversity both with our invited authors as well as within our reviewer pools. Inclusivity in science extends beyond gender, with minorities and some nationalities still underrepresented in many scientific fields. Equally, the editorial team's commitment to diversity does not end with gender as we also ensure demographic representation among our authors and reviewers. Furthermore, we encourage commissioned authors to consider diversity when deciding upon their choice of co-authors. Beyond Nature Reviews Cancer, the Nature Research journals offer doubleblinded peer review as an opt-in, in which referees do not know the identity of the authors as all relevant information is blanked out. Although difficult to hide the author identity on manuscripts, it represents one solution to counteract unconscious bias and to provide better objectivity from our reviewers.

Finally, if we are to realize gender equality, it will require all scientists, both men and women, to take responsibility and work together to find solutions. Indeed, the themes presented in these two Viewpoints are intimately linked, with one potential approach for attaining gender balance being the establishment of positive mentor-mentee relationships, irrespective of gender. By changing mindsets and providing scientists with the tools to do so, we might begin to reverse the dysfunction that has become embedded in science today.

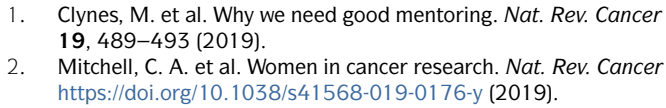

1. Clynes, M. et al. Why we need good mentoring. Nat. Rev. Cancer 19, 489-493 (2019)

2. Mitchell, C. A. et al. Women in cancer research. Nat. Rev. Cancer https://doi.org/10.1038/s41568-019-0176-y (2019).

\section{RELATED LINKS}

Nature Awards for Mentoring in Science: https://www.nature.com/nature/ awards/mentorship

Lorne Cancer conference: https://www.lornecancer.org/ 